\title{
Evaluation of Robotic Force Control Strategies using an Open Architecture Test Facility
}

\author{
Michael Short \\ University of Leicester \\ United Kingdom
}

\section{Introduction}

Industrial robots are currently employed in a large number of applications and are available with a wide range of configurations, drive systems, physical sizes and payloads. However, the numbers in service throughout the world are much less than predicted over twenty years ago (Engelberger 1980). This is despite major technological advances in related areas of computing and electronics, and the availability of fast, reliable and low-cost microprocessors and memory. This situation is mainly a result of historical and economic circumstances, rather than technical considerations. Industrial robots have traditionally performed a narrow but well-defined range of tasks to a specified degree of accuracy and whilst new robot arm designs are specified for many years of continuous operation, the technological development of their controllers has been slow in comparison with other computer-based systems.

Traditionally, most industrial robots are designed to allow accurate and repeatable control of the position and velocity of the tooling at the device's end effector. Increasingly, these systems are often also required to perform complex tasks requiring robust and stable force control strategies. In addition, task constraints sometimes require position or velocity control in some Degrees-Of-Freedom (DOF), and force control in others. Thus, to fulfil these extra demands, an important area of robotics research is the implementation of stable and accurate force control. However this is often difficult to achieve in practice, due to the technological limitations of current controllers, coupled with the demanding requirements placed upon them by the advanced control schemes that are needed in cases where robots are operating in unpredictable or disordered environments.

This chapter describes a research project that has been undertaken to partly address these issues, by investigating algorithms and controller architectures for the implementation of stable robotic force control. The chapter is organised as follows. In Section 2, the fundamental concepts of robotic force control are introduced, and the problems inherent in the design of stable, robust controllers are described. This Section also describes some of the difficulties that are faced by developers when implementing force control strategies using traditional robot controllers. It is shown that linear, fixed-gain feedback controllers designed using conventional techniques can only provide adequate performance when they are tuned to specific task requirements. In practice the environmental stiffness at the robot/task 
interface may be unknown and bounded, and may even vary significantly during the course of a specific task. In such cases, performance can be significantly degraded and is often exacerbated further by the sampling and processing limitations of traditional robot controllers.

In Section 3, a brief summary of previous work in the area of force control is given. Several strategies designed to help ameliorate the stability problems described in Section 2 are covered; two of these novel force control strategies are then discussed in greater depth. The first of these two techniques is based around an adaptive PD controller implemented using fuzzy inference techniques. The second technique centres on a model-following force controller that is robust to bounded uncertainty in the environmental stiffness. General design principles for both types of controller are discussed; the remainder of the chapter seeks to further investigate the performance of these two strategies. Section 4 describes a prototype open architecture robot controller that has been developed to overcome some of the fundamental restrictions of traditional controllers; this facility allows the direct real-time implementation of the force controllers.

Section 5 provides comparative results from a series of experiments that were undertaken to evaluate the performance of the controllers. Several additional measures of real-time performance and design complexity are also discussed. In Section 6, it is concluded that although both controllers display comparable performance, the model-based controller is favourable due to its reduced implementation overheads and reduced design effort, coupled with the fact that it lends itself to a simpler stability analysis.

\section{Robotic Force Control}

A typical conventional force control scheme is shown in Figure 1 (Zhang \& Hemami 1997; Whitney 1985; Bicker et al. 1994). In the figure, $\mathrm{f}_{\mathrm{r}}$ is the reference force, $\mathrm{f}_{\mathrm{m}}$ is the measured (processed) force, $f_{e}$ is the force feedback error and $f_{a}$ is the actual applied force. The 'Position Controlled Robot' block consists of a robot and its host (proprietary) controller. The force sensor and related control elements are typically implemented as a physically separate system from the host controller. A control signal $u$ is generated by the force controller, and effectively passed to the host controller as a vector of reference positions to be tracked. The end effector generates the forces and torques through interaction with the current contact dynamics. When implementing such a strategy, it is common for the external outer loop controller to pass the position commands to the proprietary joint controller over some form of communications link; such a feature has been common in most industrial robot controllers for many years. For example the ALTER command with the PUMA range of robots allows position setpoints to be sent from an external device over an RS-232 serial link, using a simple messaging protocol (Bicker et al. 1994).

The contact dynamics are represented by the combined stiffness at the end effector/task interface in the direction of the applied force $\left(\mathrm{K}_{\mathrm{e}}\right)$. There is quite often a very short lag in these dynamics; however this is often neglected as it is many orders of magnitude smaller than the dominating lags elsewhere in the system. The environmental stiffness gain typically varies between a minimum value, determined by the objects in the environment with which the robot is in contact, and a maximum value, limited by the stiffness of the arm and torque sensor. The latter is dominant when the robot is touching a surface of very high stiffness, i.e. in a hard contact situation. Designing a fixed-gain conventional controller to meet a chosen 
specification for a specific value of $\mathrm{K}_{\mathrm{e}}$ is, in principle, a relatively straightforward task. A problem arises when $K_{e}$ is unknown or variable; for example, consider the case where the system is tuned to achieve a specified performance at an upper limit of $K_{e}$. At low $K_{e}$ the system will be overdamped, with a relatively high settling time. Conversely, if the system has been tuned for the desired performance at the lower limit of $\mathrm{K}_{\mathrm{e}}$, significant overshoot and oscillatory behaviour would occur at higher stiffness values. Figure 2 shows such a situation, using data recorded for the robotic system described in Section 4. In this figure, two plots of contact force for a fixed-gain controller tuned for low $\mathrm{K}_{\mathrm{e}}$ are displayed. The low $\mathrm{K}_{\mathrm{e}}$ contact situation is as expected; however oscillatory behaviour for the high $\mathrm{K}_{\mathrm{e}}$ situation can clearly be seen. In practical robotic systems, this kind of 'chattering' behaviour can have serious consequences, potentially causing serious damage to the robot and its environment.

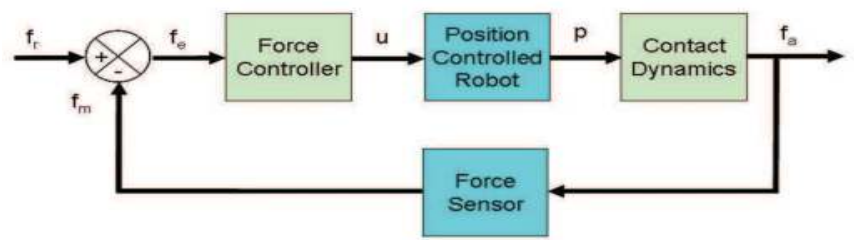

Fig. 1. Typical conventional robotic force control scheme

Other major factors contributing to poor, unstable performance include the finite and relatively low sampling rates of many industrial robot control systems. These problems are often considerably worsened by the presence of noise, non-linearities and other factors. For this reason, force controllers of the type described usually require some form of environment stiffness detection technique to enable the controller gains to be switched accordingly. The main problem with this process is that it is time consuming, often involving 'guarded moves' to contact in order to enable sufficient data to be collected for the algorithm to work. Such methods are also vulnerable to the presence of transducer noise, and are not very effective in situations where $K_{e}$ is variable or rapidly changing - for example during a deburring task (Ow 1997). This also has the effect of slowing down task execution significantly. Problems such as these have motivated much research into designing efficient force control schemes, and this is the subject of the next Section.

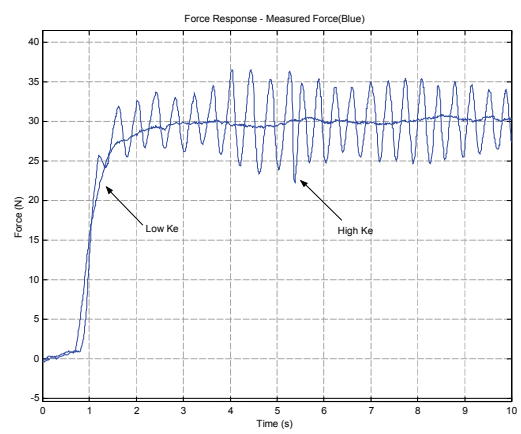

Fig. 2. Environmental stiffness effects on the performance of a fixed-gain force controller 


\section{Advanced Force Control Schemes}

A large number of force control techniques of varying complexity have been proposed over the last twenty years (Zhang \& Hemami 1997; Whitney 1985). The most basic direct methods simply transform joint-space torques into a Cartesian-space wrench, either in an open-loop fashion (which does not require the explicit measurement of forces and torques) or using inner and outer closed loops for accurate control of joint torques and Cartesian forces, respectively. However, since most industrial robots have position control loops that are not easily modified, indirect methods such as those described in the previous Section are often preferred. As mentioned, these involve modifying either joint or Cartesian position setpoints in order to control forces by deliberately introducing position control errors and using the inherent stiffness of the manipulator in different Cartesian directions.

As mentioned, stable force control is particularly difficult to achieve in 'hard' or 'stiff' contact situations, where the control loop sampling rate may be a limiting factor. In an attempt to improve stability various methods have been proposed, the simplest being the addition of compliant devices at the robot wrist (Whitney \& Nevins 1979). Another solution is to employ 'active compliance' filters, where force feedback data is digitally filtered to emulate a passive spring/damper arrangement (Kim et al. 1992). However, both methods introduce a potentially unacceptable lag. Recent increases in processing power of low-cost computers has led to an increased interest in 'intelligent control' techniques such as those employing fuzzy logic, artificial neural networks and genetic algorithms (Linkens \& Nyongsa 1996). Where attempts have been made to employ these techniques (specifically fuzzy logic) in explicit robot force controllers, simulation studies have demonstrated good tracking performance despite wide variations in environment stiffness, e.g. (Tarokh \& Bailey 1997; Seraji 1998), and for specific contact situations, e.g. deburring (Kiguchi \& Fukuda 1997). Improved performance using a hierarchical fuzzy force control strategy has also been demonstrated for various contact situations, such as peg-in-hole insertion (Lin \& Huang 1998). A highly successful and generically applicable force control strategy based upon a Sugeno-style Fuzzy Inference System (FIS) was proposed by Burn et al. (2003), and will be described in more detail in Section 3.1.

However, these fuzzy techniques are not without problems. In addition to problems associated with the 'curse of dimensionality', i.e. large numbers of rules that must be evaluated in the inference process, the performance and stability of fuzzy systems are often difficult to validate analytically (Cao et al. 1998; Wolkenhauer \& Edmunds 1997). Additionally, when compared to more 'traditional' control methods such as LQR (Frankin et al. 1994), the resulting fuzzy designs are more complex, have larger memory requirements and larger execution times (Bautista \& Pont 2006). Such a technique which has proved to be popular in recent years has been the use of Model Following Control (MFC). Due to its conceptually simple design and powerful robustness properties, this type of controller has been found to be particularly suited to industrial applications such as robotics and motion control (e.g. Li et al. 1998; Osypiuk et al. 2004). Recent investigations have also shown that MFC-based techniques can be successfully applied in the force control domain (Short \& Burn 2007). The MFC-based force control technique will be investigated further in Section 3.2.

\subsection{Fuzzy Approach To Force Control}

A method of designing Sugeno-style fuzzy controllers has previously been developed that 
effectively produces a Proportional + Velocity (PV) controller with variable gains, capable of maintaining acceptable performance irrespective of $\mathrm{K}_{\mathrm{e}}$ (Burn et al. 2003). A block diagram of the arrangement is shown in figure 3. To design a controller using this method, firstly a Sugeno-style FIS is created to emulate a conventional PV controller tuned for a high $\mathrm{K}_{\mathrm{e}}$ environment. The FIS is assigned three inputs $\left(\mathrm{f}_{\mathrm{e}}, \Delta \mathrm{f}_{\mathrm{e}}\right.$ and $\left.\Delta \mathrm{p}\right)$, and one output $(\mathrm{u})$, where the input ranges are measured from conventional system data. The output from the FIS is a velocity demand. In order to create a linear system, initially only a single Membership Function (MF) for each input and output is required. By assigning names normal to the input MF's, and $\mathrm{u}_{1}$ to the output MF, a rule of the following form produces the desired linear control surface: IF $\left(\mathrm{f}_{\mathrm{e}}, \Delta \mathrm{f}_{\mathrm{e}}, \Delta_{\mathrm{p}}\right)$ are 'normal' then $\mathrm{u}$ is $\mathrm{u}_{1}$. Note that a consequence of employing only one rule is that no defuzzification algorithm is required. By employing a first-order, Sugeno-style FIS, output $\mathrm{u}_{1}$ is then defined by:

$$
u_{1}=K_{1} \cdot f_{e}+K_{2} \cdot \Delta f_{e}+K_{3} \cdot \Delta p+K_{4}
$$

where $\mathrm{K} 1$ is a positive constant (equal to the forward gain $\mathrm{K}_{\mathrm{p}}$ of a PV controller), $\mathrm{K} 3 \mathrm{a}$ negative constant (equal to the velocity feedback gain $\mathrm{K}_{\mathrm{v}}$ ), and $\mathrm{K} 2$ and $\mathrm{K} 4$ are - in this case set to zero.

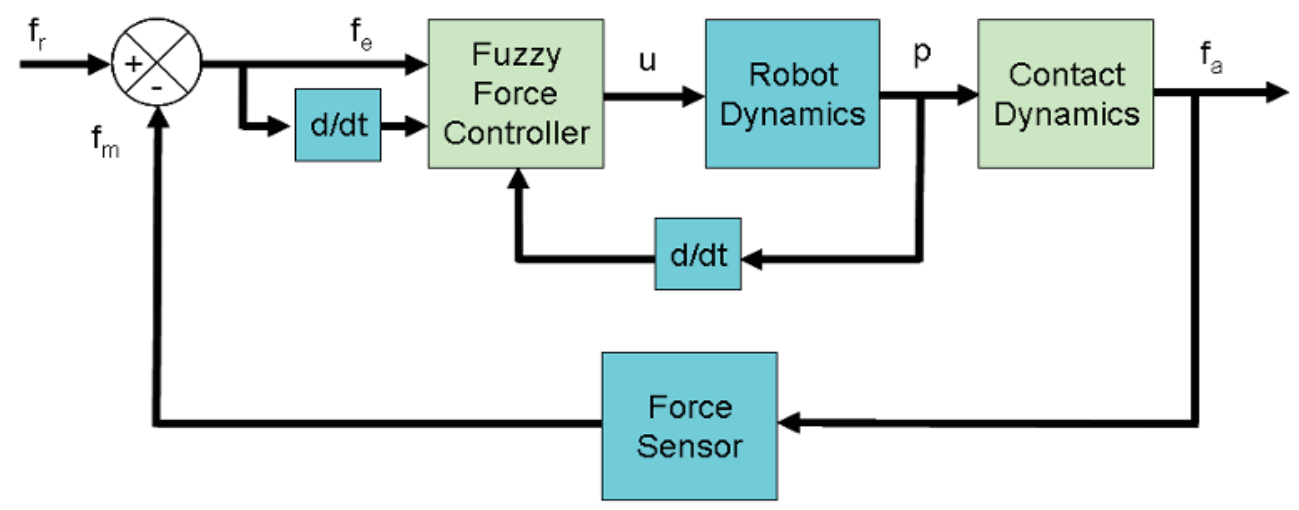

Fig. 3. Fuzzy force controller

The choice of MF type is influenced by the concept of data 'spread', and the measurement or calculation of standard deviation data oi from step response tests. For the single rule system each input is assigned a single Gaussian MF centered at zero, each with a $\sigma_{\text {normal }}$ parameter equal to that of data obtained from tuned step responses at high $\mathrm{K}_{\mathrm{e}}$. Since the single rule system emulates a conventional PV controller it suffers the same disadvantages in the face of variable $\mathrm{K}_{\mathrm{e}}$. However, having created the initial FIS, it is now possible to modify the controller using a combination of analytical and intuitive methods.

With the system tuned for high $\mathrm{K}_{\mathrm{e}}$, during soft contact the maximum value of $\Delta \mathrm{f}_{\mathrm{e}}$ is reduced. This reflects an overdamped response, an undesirable effect that can be minimized by increasing the proportional gain component of the controller output given by equation (1) if 
lower $\Delta \mathrm{f}_{\mathrm{e}}$ is 'detected' by the fuzzy controller. This is achieved initially by adding a second Gaussian $M F$ to the $\Delta \mathrm{f}_{\mathrm{e}}$ input set (low), with a smaller standard deviation $\sigma_{\Delta \text { felow }}$. In addition, during a dynamic response of a tuned system to a step input, the maximum value of $\Delta \mathrm{p}$ is inversely proportional to $\mathrm{K}_{\mathrm{e}}$. In other words, $\Delta \mathrm{p}$ increases during soft contact. A second rule is thus added to take into account the decrease in $\Delta \mathrm{f}_{\mathrm{e}}$ relative to the 'normal' (desired) profile, and the relative increase in $\Delta \mathrm{p}$. By adding a second output of the same form as equation (1) it is possible to vary the effective gains. Therefore, a rule is added of the form: $\operatorname{IF}\left(\Delta \mathrm{f}_{\mathrm{e}}\right.$ is low) AND ( $\Delta \mathrm{p}$ is high) then $\mathrm{u}$ is $\mathrm{u}_{2}$, where $\mathrm{u}_{2}$ has the same form as $\mathrm{u}_{1}$ in equation (1), but with a modified forward gain component $K 1_{a}$, equivalent to $K_{p}$ tuned for soft contact such that $\mathrm{K} 1_{\mathrm{a}}>\mathrm{K} 1$, and $\sigma \Delta \mathrm{p}_{\text {high }}>\sigma \Delta \mathrm{p}_{\text {normal }}$.

The advantage of the method lies in its apparent simplicity, although its success relies upon the correct determination of the MF parameters, particularly $\sigma \Delta \mathrm{p}_{\text {high }}$ and $\sigma \Delta \mathrm{p}_{\text {normal. }}$. Due to the structured and well-defined methodology utilized in creating the controller design, as a related work a software design tool was created that automates the process of designing a fuzzy force controller. The tool includes an iterative method to tune these MF parameters until acceptable performance is achieved (Burn et al. 2004).

\subsection{Model-Based Approach To Force Control}

The robust model-based force controller previously described by Short \& Burn (2007) is loosely based around a robust PID strategy discussed in detail by Scokzowski et al. (2005). The original strategy is based upon a two-loop MFC, containing a nominal model of the controlled plant and two PID controllers. The block diagram of a basic MFC controller is shown in figure 4 .

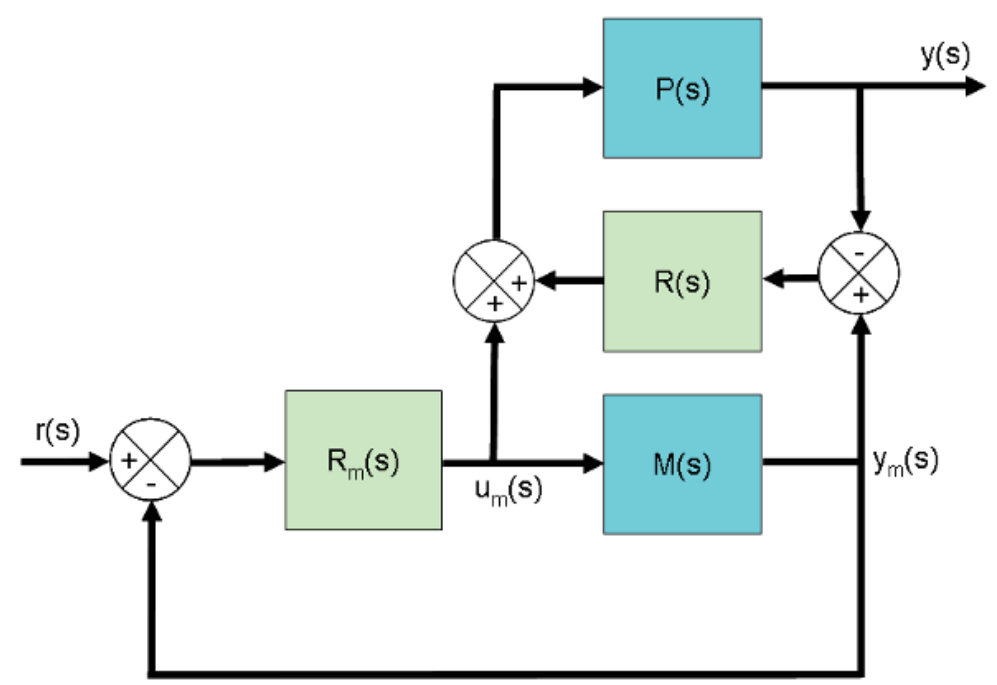

Fig. 4. Robust PID based on MFC

In this type of control, the model compensator $R_{m}(s)$ is tuned to a nominal model of the plant $\mathrm{M}(\mathrm{s})$; the actual plant $\mathrm{P}(\mathrm{s})$ contains bounded uncertainties. The auxiliary controller 
$\mathrm{R}(\mathrm{s})$ acts on the difference between the actual process output and the model process output to modify the model control signal $\mathrm{u}_{\mathrm{m}}(\mathrm{s})$, which is also fed to the plant. In the case of robotic force control, the model $\mathrm{M}(\mathrm{s})$ is simply the second order motion control loop dynamics, augmented by a free integrator, and a known (base) value of environment stiffness.

Assuming that model is of reasonable quality, the bounded uncertainty in the plant is then dominated by the environment stiffness $K_{e}$, varying between $K_{e m a x}$ and $K_{e m i n}$.

If the two loop controllers $\mathrm{R}(\mathrm{s})$ and $\mathrm{R}_{\mathrm{m}}(\mathrm{s})$ are simple proportional gains, as shown in Figure 5 , then the MFC structure is considerably simplified. The model loop gain $K_{p}$ can be tuned for $K_{\text {emax }}$ - a relatively trivial task - whilst the auxiliary loop gain $K_{p}{ }^{\prime}$ can be tuned to provide an additional control signal should the actual value of $K_{e}$ be less than $K_{e m a x}$. However, with this type of controller structure it is important to consider the stability criteria, and provide a bound on the maximum value for $\mathrm{K}_{\mathrm{p}}^{\prime}$.

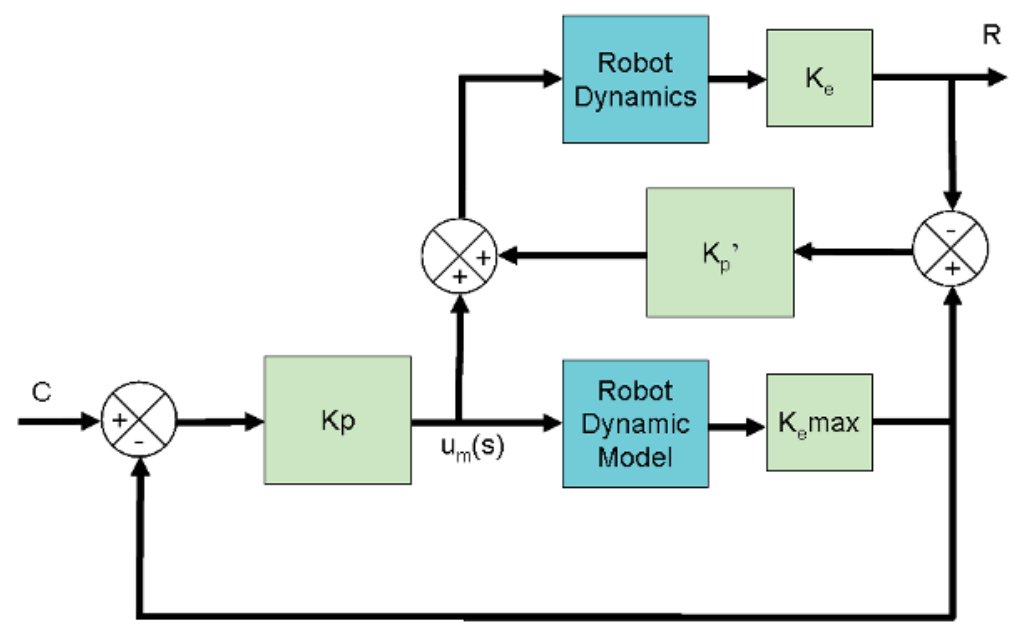

Fig. 5. Robust force controller

If the 'model loop' controller $\mathrm{R}_{\mathrm{m}}(\mathrm{s})$ is tuned for stability using a nominal design method on the plant $\mathrm{P}(\mathrm{s})$ augmented by the maximum environmental stiffness gain $\mathrm{K}_{\mathrm{emax}}$, then the stability of the overall control strategy is restricted by the roots of the equation:

$$
1+R(s) M(s)[1+\Delta(s)]=0
$$

Where $\Delta(\mathrm{s})$ denotes the model perturbations (uncertainty). The objective is to find for a given plant and bounded uncertainty in the stiffness gain a maximum bound on $|R(s)|$ that will maintain stability. In the case where the uncertainty exclusively resides in the environment stiffness gain $K_{e}$, then if the original loop is tuned for $K_{e m a x}$ then $M(s)[1+\Delta(s)]$ in (2) reduces to:

$$
M(s)[1+\Delta(s)]=P(s)=G(s) K_{e \max }
$$


Where $G(s)$ represents the nominal robot dynamics and has the form (due to the free integrator in the forward path):

$$
G(s)=\frac{\omega_{n}{ }^{2}}{s^{3}+2 \xi \omega_{n} s^{2}+\omega_{n}{ }^{2} s}
$$

Since the controller $R(s)$ in this case is a single gain, $K_{p}{ }^{\prime}$, using (3) and (4), equation (2) can be re-written as follows:

$$
s^{3}+2 \xi \omega_{n} s^{2}+\omega_{n}^{2} s+\omega_{n}^{2} K p^{\prime} K e_{\max }=0
$$

Applying the Routh-Hurwitz stability criterion (Pippard 1997) for a cubic equation, the system will be stable if all the co-efficients in the left of (5) are positive, and the following criterion is satisfied:

$$
2 \xi \omega_{n} \omega_{n}^{2} \geq \omega_{n}^{2} K p^{\prime} K e_{\max }
$$

Re-arranging (6) gives a stability limit for the controller gain $\mathrm{K}_{\mathrm{p}}{ }^{\prime} \max$ as follows:

$$
K p_{\max }^{\prime}=\frac{2 \xi \omega_{n}}{K e_{\max }}
$$

Thus if the gain $\mathrm{K}_{\mathrm{p}}{ }^{\prime}$ is chosen between the limits:

$$
K p<K p<K p_{\max }
$$

The controller will be stable for unknown environment gains in the range $0<\mathrm{K}_{\mathrm{e}} \leq \mathrm{K}_{\mathrm{emax}}$; as for all gains below $\mathrm{K}_{\mathrm{emax}}$, the stability criteria of (6) holds. Clearly, the formulation of these two controllers follows two distinct paths. The first is mainly based on an intuitive, heuristic formulation, while the second is based on a more thorough analytical approach. In Section 5, experimental results are presented for both controllers applied to an experimental test facility, which is described in the following Section.

\section{Experimental Test Facility}

\subsection{Description}

A research facility, previously described in detail (Burn \& Short 2000; Short 2003), has been developed in the form of a planar robot arm and PC-based open architecture controller. The 
robots joints are manufactured from toughened ABS plastic, and are actuated by brushless servomotors (with digital servoamplifiers). The control loop for each axis is closed via a multitasking DSP embedded in a Delta Tau ${ }^{\circledR}$ Programmable Multi-Axis Controller (PMAC) motion control card, installed into the PC. Each axis has an individual PID controller with feed forward control to enable accurate velocity and position profile following. A six-axis force/torque sensor was developed in-house for the project, and is employed in the current study. The robot arm is shown photographically in figure 6, and schematically in figure 7.

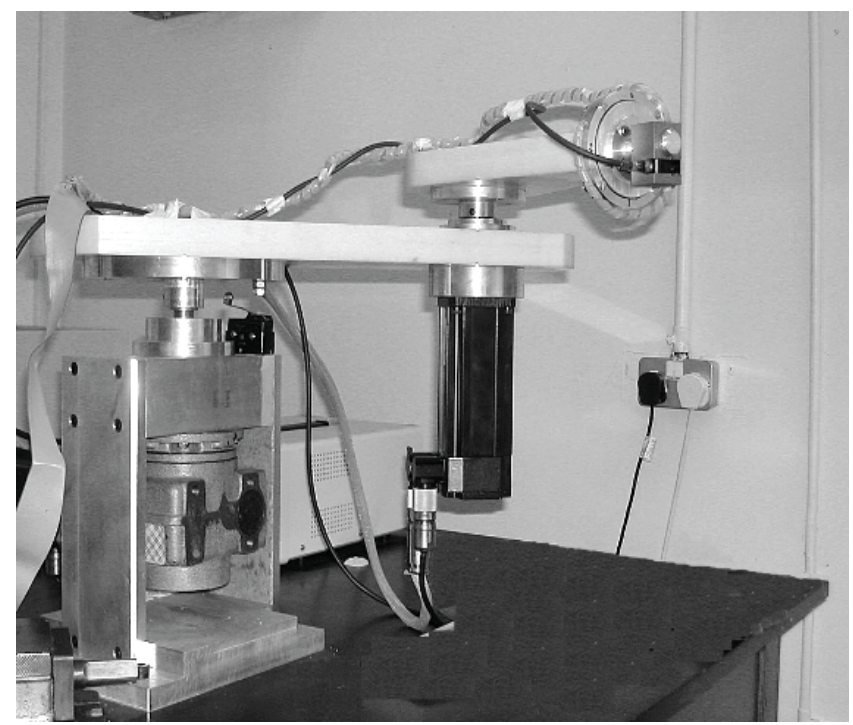

Fig. 6. Robotic test facility

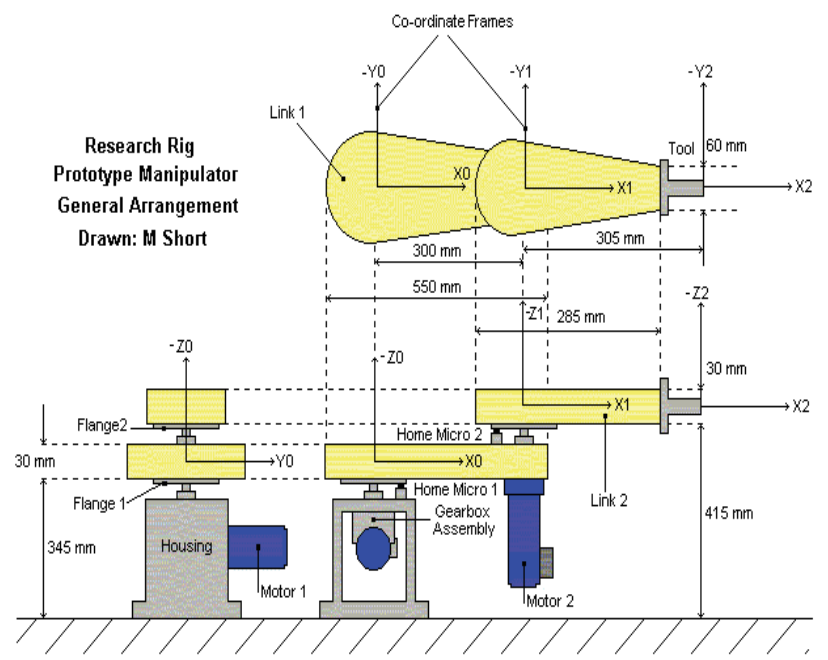

Fig. 7. Schematic of test facility 


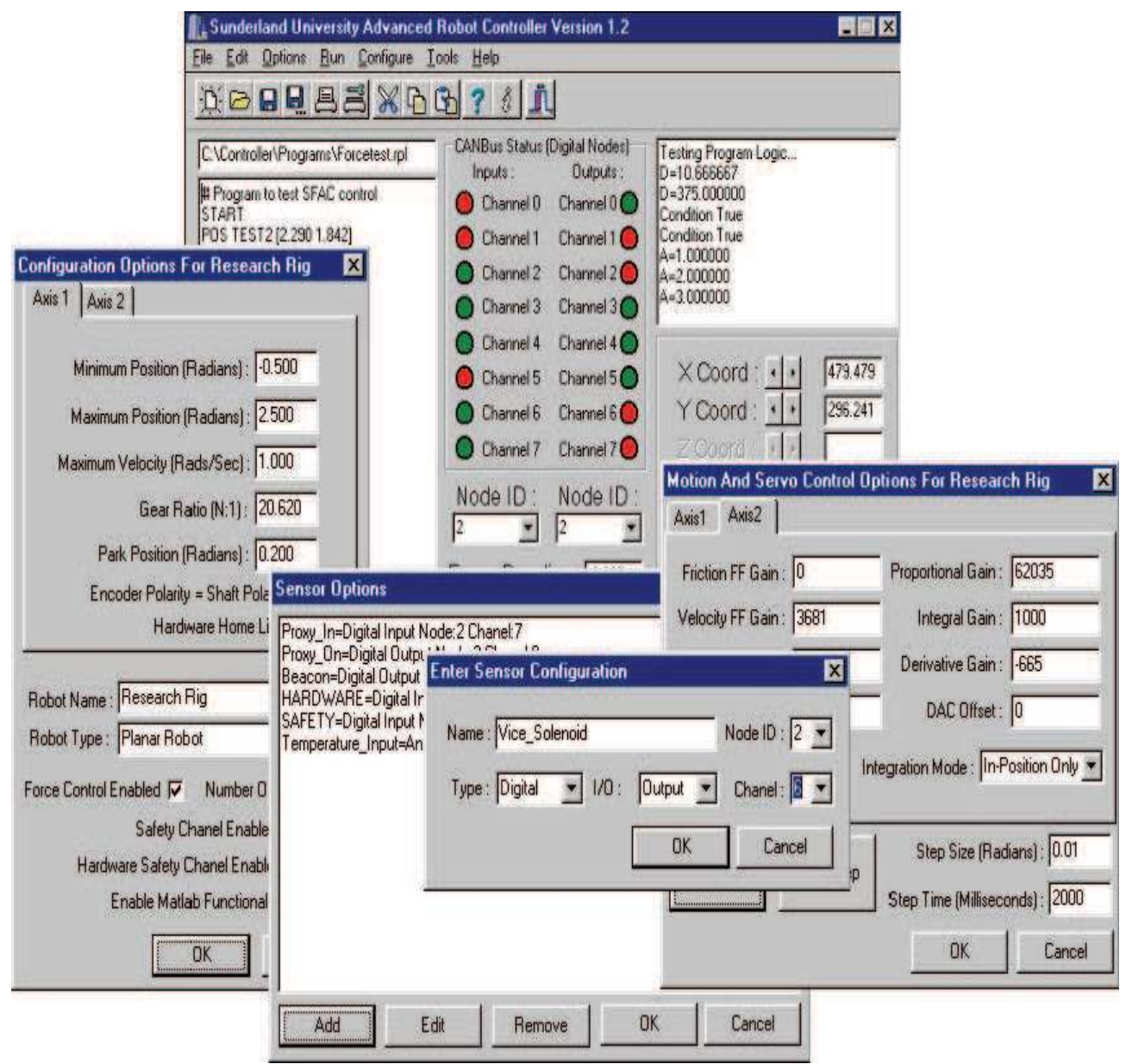

Fig. 8. Application software screenshots

The controller for this robot was developed with a completely 'open architecture' in mind (Ford 1984), with a view to the integration and implementation of novel sensor-based control strategies. The software is based on a three-layered open architecture, as described by Short ( $2000 \& 2003)$. It features the ability to design and integrate advanced control strategies into the controller, and via the use of an ActiveX® link allows the full functionality of software packages such as Matlab® to be embedded within the controller software. The sensors required to perform these control strategies in real-time are integrated into the system using flexible fieldbus technologies. The underlying kinematic and dynamic models of the robot can be changed to suit the current configuration, allowing the controller to be tailored to any particular arm configuration or drive system. A modular robot programming language - named Sunderland ARm Language (SARL) - was developed for 
the controller. Screenshots of the Windows ${ }^{\circledR}$ XP version of the application software are shown in figure 8 .

The force controllers described in the previous Section were coded in the C programming language, compiled and added into the controller's modular software component library. Several experiments were then performed using the resulting software. Each experiment involved a specific contact situation, where the robot first approached the contact surface with constant velocity, and subsequently applied a force of $30 \mathrm{~N}$. The contact surface was varied in each experiment, and two surfaces were used; hard (steel) and soft (plastic). In order to reliably detect the contact surface, the end effector was fitted with a Baumer Electric ${ }^{\circledR}$ photoswitch which was calibrated to signal with high accuracy when a solid object was within a distance of $5 \mathrm{~mm}$. The sensor was integrated into the controller architecture using a Controller Area Network connection (CAN) fieldbus. The robot was programmed approached the contact surface at a slow jog speed until this signal was made, then switched to force control mode. The sample rate employed was $200 \mathrm{~Hz}$ in each experiment, and the measured force was prefiltered using a first-order low pass filter with cut off frequency of $0.01 \mathrm{~Hz}$. In the following section, the parameters that were used to design the controllers are described.

\subsection{Controller Design}

From a previous identification exercise, the model parameters of each joint of the robot arm and the environment stiffness limits were determined to be as follows (Short 2003):

$$
\begin{aligned}
& \omega_{n}=244 \mathrm{rad} / \mathrm{s}, \xi=1, \\
& K_{e \max }=168 \mathrm{~N} / \mathrm{mm}, \quad K_{e \text { min }}=11 \mathrm{~N} / \mathrm{mm}
\end{aligned}
$$

Using these parameters, the controllers were designed as follows. In both cases, the nominal loop gain $K_{p}$ was tuned to a value of 0.02 to give the desired transient performance - a $95 \%$ rise time of approximately 2 seconds with minimal overshoot. For the fuzzy method, the nominal gain for contact at low $\mathrm{K}_{\mathrm{e}}$ could then simply be calculated as follows:

$$
K_{\text {plow }}=\left(\frac{K_{e \max }}{K_{e \min }}\right) \cdot K_{p}=0.3
$$

This information was then used as input to the controller design software (Burn et al. 2004), and the controller tuning algorithm was run for 200 iterations to produce the final fuzzy controller that was utilized in the experiment. The highly non-linear I/O surface of the controller is illustrated by figure 9, which shows a plot of $\mathrm{F}_{\mathrm{e}}$ and $\Delta \mathrm{F}_{\mathrm{e}}$ versus controller output $\mathrm{u}$. In the MFC controller, the value of the nominal loop gain $\mathrm{K}_{\mathrm{p}}$ was then used to design the value of $\mathrm{K}_{\mathrm{p}}{ }^{\prime}$ max calculated as given by (7) to have a value of 2.9. A value of $\mathrm{K}_{\mathrm{p}}{ }^{\prime}=$ 1.5 was therefore chosen for the experiments, as this gave good performance and remained well below the stability limit. The experimental comparison of the controllers is discussed in the following Section. 


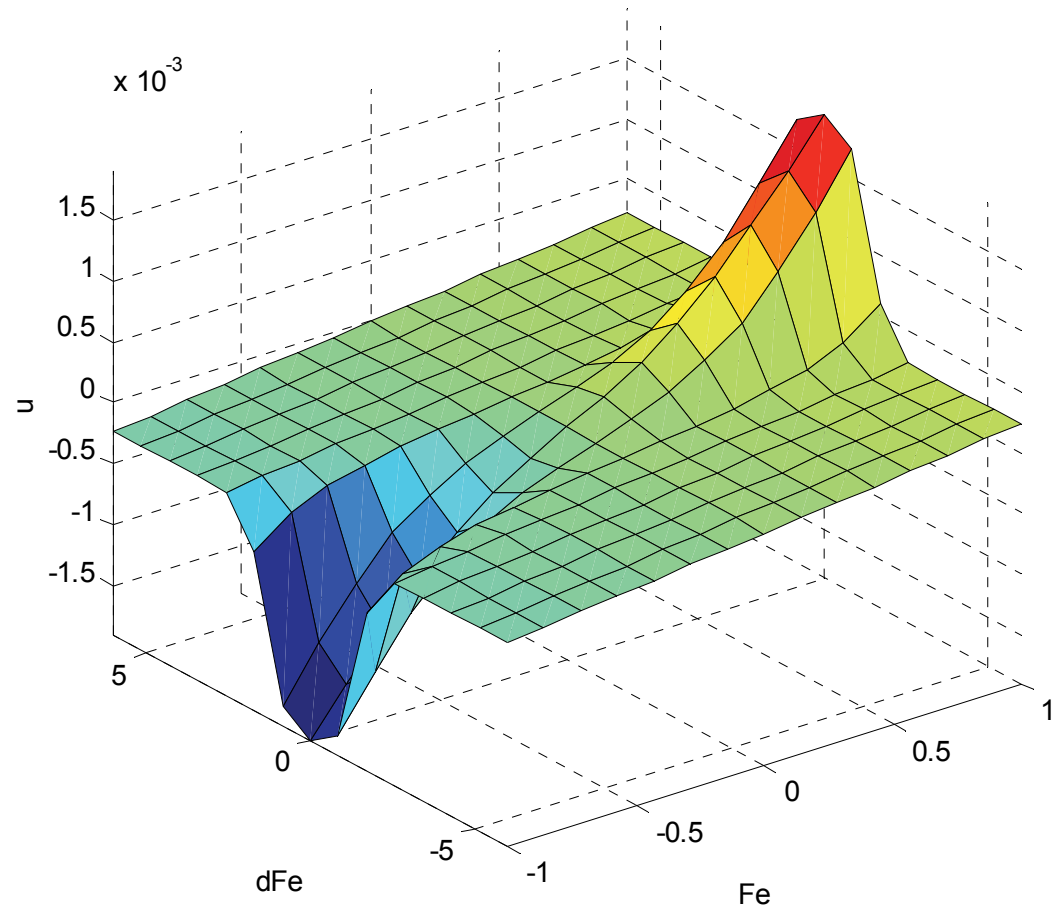

Fig. 9. Fuzzy controller I/O surface

\section{Experimental Results and Analysis}

This section begins by presenting the results of the contact experiments described in the previous Section, beginning with the FIS-based controller. Figure 10 shows the responses of this controller when applying a force to the hard (steel) and soft (plastic) surfaces. Considering now the MFC-based controller, figure 11 shows the responses of this controller when applying a force to the same surfaces. The very small negative force indicated before contact with the surface was made (at approx 1s) was due to a small drift in the calibration of the force sensor whilst moving in free space.

These figures demonstrate the effectiveness of both approaches; comparing these figures with the responses shown in figure 2, the fixed gain controller, it can be seen that the responses display little sign of instability. The compensation added by the adaptation of loop gains in the FIS controller, and the extra loop and forcing gain in the MFC controller can clearly be seen; in all cases, a very similar transient response is seen. There is a slight overshoot in the response of the fuzzy method when contacting the hard surface, and it can be seen that the steady-state behaviour seems to be slightly less stable than the MFC controller. 


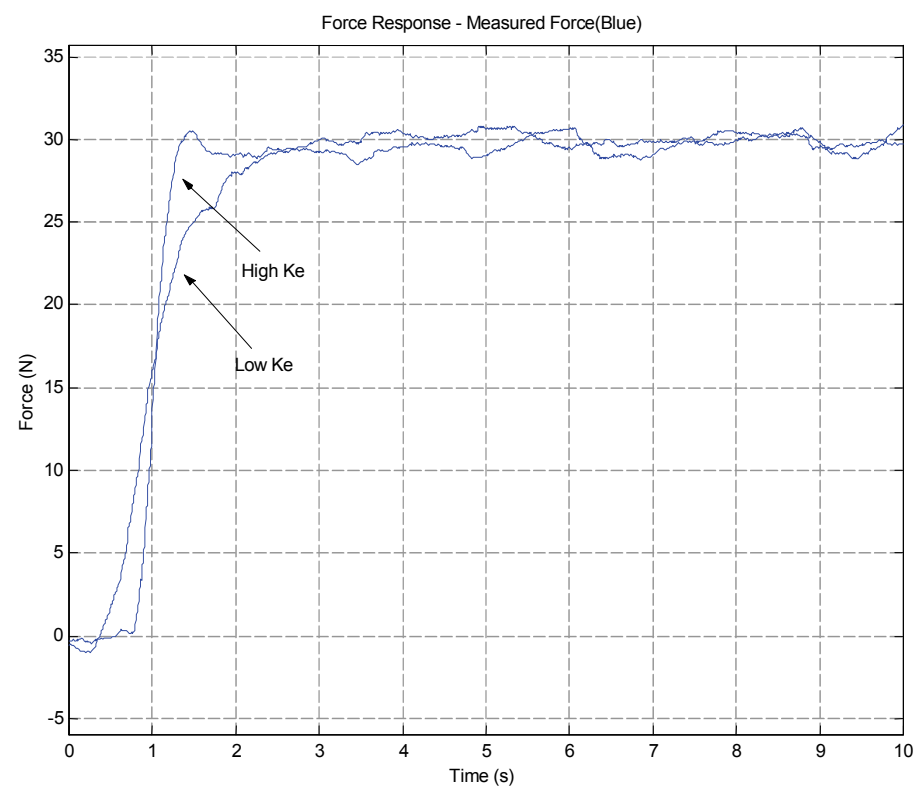

Fig. 10. Contact force profile for the FIS-based controller

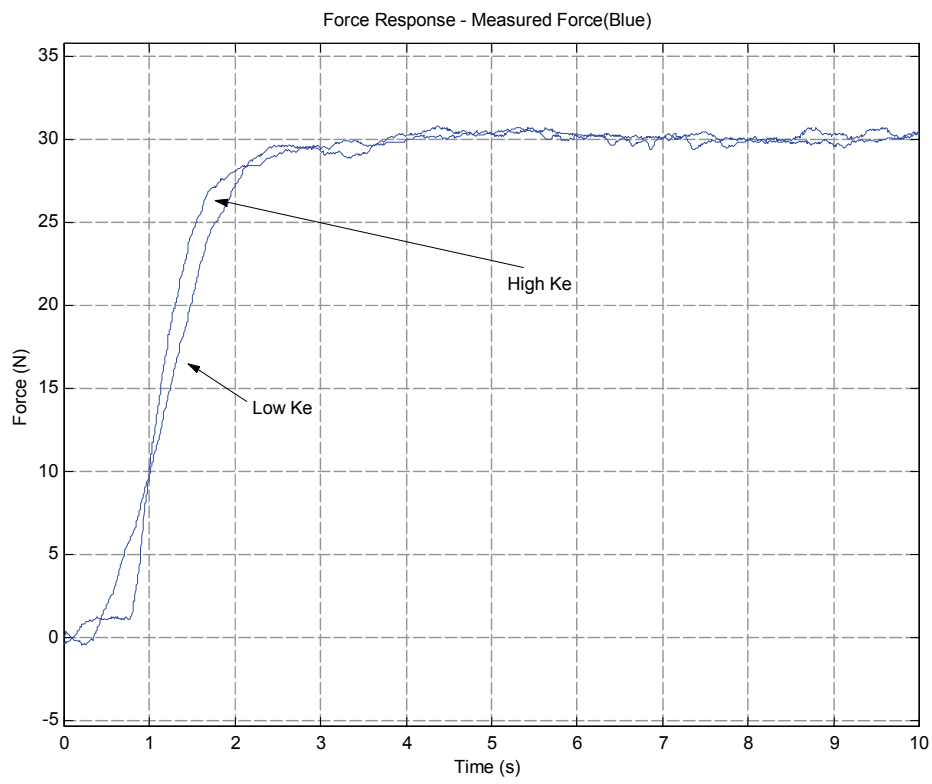

Fig. 11. Contact force profile for the MFC-based controller 
In addition to these response measurements, the Integral of Time by Absolute Error (ITAE) for each of the responses was calculated and is shown in table 1. The ITAE is a useful measure of system performance in the time-domain and is given by equation (11) (Franklin et al. 1994). This table includes additional information pertaining to each method, including the approximate Source-Lines-Of-Code (SLOC) needed for each implementation, the measured run-time overheads (i.e. CPU execution time per iteration, in milliseconds) and a relative measure of the 'design effort' needed for each controller. The latter was classified subjectively into either a LOW or HIGH category.

$$
\text { ITAE }=\int|e| \cdot t . d t
$$

\begin{tabular}{|c|c|c|c|c|c|}
\cline { 2 - 6 } \multicolumn{1}{c|}{} & \multicolumn{5}{c|}{ Performance Measures } \\
\hline Controller & ITAE (low) & ITAE (high) & SLOC & CPU (ms) & Effort \\
\hline FIS & 227.1 & 247.1 & $1000+$ & 2.05 & HIGH \\
\hline MFC & 215.6 & 220.1 & $<100$ & 0.15 & LOW \\
\hline
\end{tabular}

Table 1. Summary of controller comparative data

The ITAE data further illustrates the performance of both force control methods. As expected, the FIS controller had a higher overall ITAE value for both contact situations, and there was a larger measurable difference between the two response values. This reflects the observation that the FIS controller demonstrated a faster response with a slight overshoot at the higher stiffness gain value. Although a slight difference exists between the responses for the MFC controller, as can be seen from the responses its performance seems more predictable and it does not 'hunt' around the setpoint to the same level as the FIS. This is best attributed to the fact that close to the setpoint, the model error - and hence the forcing control signal - are close to zero. The FIS controller, however, seems to be sensitive to noise in the error signal; especially through the $\Delta \mathrm{f}_{\mathrm{e}}$ input, thus allowing small gain adaptations to take place in the region of the setpoint. These small gain adaptations are magnified by the resulting changes in the measured $\Delta_{\mathrm{p}}$, exacerbating the problem slightly. Despite these observations, it can be argued that the overall performance of both controllers is acceptable for most practical situations.

The SLOC measure gives a rough indication of the complexity of the code required for the implementation of the controllers. In this respect it can be seen that the MFC has a huge advantage over the FIS. As well as the main implementation code, e.g. the calculation of error signals for the controller inputs, the FIS method requires extensive code to implement the fuzzification, logical inference and de-fuzzification subroutines. These will - generally cause a large increase in the code size, either from the inclusion of specialised library files or from a direct implementation of the underlying mathematical equations. The MFC controller, however, simply requires the updating of a third-order equation and simple addition/subtraction and multiplication to generate the control effort. The increased complexity of the FIS method is reflected by an almost 14-fold increase in the CPU 
overheads required at each sample iteration to generate the control signal. In addition, although the design methodology proposed for the MFC controller guarantees its stability at present, no such guarantee can be placed on the FIS-based controller. To summarize, it was reflected that the amount of effort required to implement the FIS method was significantly higher than the MFC, from both the control and software design perspectives.

\section{Conclusion}

This chapter has been concerned with the practical realisation of robotic force control. It has been shown that many potential difficulties arise when implementing a force control method, including stability and robustness problems associated with applications where environmental uncertainty exists, and with sampling and control limitations related to the basic operation of many tradition robot controllers.

Force control remains an ongoing area of research; however, in recent years a variety of efficient solutions to many of these problems have been proposed. This chapter has considered two novel methods in depth, and has described a series of experiments to perform a direct real-time comparison of the controllers using an open-architecture test facility. Whilst the results generated indicate slightly better behaviour for the MFC-based method, in practical situations both methods were deemed acceptable and a considerable improvement over a fixed-gain controller. Based on the analysis of design effort, system stability and incurred CPU overheads, however, this chapter concludes that the MFC-based controller has significant advantages over the FIS-based design.

Future work in this area will include analysis of situations where PD controllers are used as the MFC loop compensators, and also consider the effects of model mismatch (which is inevitable if the methodology is to be applied to larger-scale industrial robots). Further work will also consider implementation on both controllers on a 6-DOF manipulator to further investigate and contrast the two approaches.

\section{References}

Bautista, R. \& Pont, M.J. (2006). Is fuzzy logic a practical choice in resource-constrained embedded control systems implemented using general-purpose microcontrollers? In Proceedings of the 9th IEEE International Workshop on Advanced Motion Control, Istanbul, Vol. 2, pp.692-697.

Bicker, R., Burn, K., Glennie, D. \& Ow, S.M. (1994). Application of force control in telerobotics, Proc Int Conf EURISCON ' 94, Malaga, Spain.

Burn, K, Home, G, Short, M. \& Bicker, R. (2004). A software tool for automating the design of robot fuzzy force controllers, Robotica, Vol. 23(2), pp. 247-256.

Burn, K, Short, M. \& Bicker, R. (2003). Adaptive and nonlinear fuzzy force control techniques applied to robots operating in uncertain environments, Journal of Robotic Systems, Vol. 20(7), pp. 391-400.

Burn, K. \& Short, M. (2000). Development of a generic robot controller architecture for advanced and intelligent robots, In: Proc. 14th Int. Conf. On Systems Eng. (ICSE 2000), Vol. 1, pp. 92-97.

Cao, S.G., Rees, N.W. \& Feng, G. (1998). Lyapunov-like stability theorems for continuoustime fuzzy control systems. Int J Control, Vol. 69(1), pp. 49-64. 
Engelberger, JF. (1980). Robots in practice, Kogan Page; London, UK.

Ford, WE. (1984). What is an open architecture robot controller? In: Proc 9th IEEE Int. Symp. on Intelligent Control, pp. 27-32.

Franklin, G.F., Powell, J.D. \& Emani-Naeini, A. (1994). Feedback Control Of Dynamic Systems, Addison-Wesley Publishing, Reading Massachusetts, third edition.

Kiguchi, K. \& Fukuda, T. (1997). Intelligent position/force controller for industrial robot manipulators - application of fuzzy neural networks, IEEE Trans Industrial Electronics, Vol. 44(6), pp. 753-761.

Kim, W.S., Hannaford, B. \& Bejczy, A.K. (1992). Force Reflection and Shared Compliant Control in Operating Telemanipulators with Time Delay, IEEE Trans on Robotics and Automation, Vol. 8(2), pp. 176-185.

Li, G., Tsang, K.M. \& Ho, S.L. (1998). A novel model following scheme with simple structure for electrical position servo systems, Int. J. Syst. Sci., Vol. 29(9), pp. 959-969.

Lin, S.T. \& Huang, A.K. (1998). Hierarchical Fuzzy Force Control for Industrial Robots, IEEE Trans on Industrial Electronics, Vol. 45(4), pp. 646-653.

Linkens, D.H. \& Nyongesa, H.O. (1996). Learning systems in intelligent control: an appraisal of fuzzy, neural and genetic algorithm control applications, IEE Proc Control Theory Appl, Vol. 143(4), pp. 367-386.

Osypiuk, R., Finkemeyer, B. \& Wahl, F.M. (2004). Forward-model based control system for robot manipulators, Robotica, Vol. 22(2), pp. 155-161.

Ow, S.M. (1997). Force Control in Telerobotics, PhD Thesis, University of Newcastle upon Tyne, UK.

Pippard, A.B. (1997). Response E Stability: An Introduction to the Physical Theory, Cambridge University Press.

Seraji, H. (1998). Nonlinear and Adaptive Control of Force and Compliance in Manipulators, Int J Robotics Research, Vol. 17(5) pp. 467-484.

Short, M. (2003). A Generic Controller Architecture for Advanced and Intelligent Robots, PhD. Thesis, University of Sunderland, UK.

Short, M. \& Burn, K. (2007). Robust and Stable Robotic Force Control, In: Proceedings of the 4th International Conference on Informatics in Control, Automation and Robotics (ICINCO' 07), Angers, France, May 09-12.

Skoczowski, S., Domek, S., Pietrusewicz, K. \& Broel-Plater, B. (2005). A Method for Improving the Robustness of PID Control, IEEE Trans Industrial Electronics, Vol. 52(6), pp. 1669-1676.

Tarokh, M. \& Bailey, S. (1997). Adaptive fuzzy force control of manipulators with unknown environment parameters, J Robotic Sys, Vol. 14(5), pp. 341-353.

Whitney, D.E. (1985). Historical Perspective and State of the Art in Robot Force Control, Int J Robotics Res, Vol. 6(1), pp. 3-14.

Whitney, D.E. \& Nevins, J.L. (1979). What is the Remote Centre Compliance (RCC) and what can it do? In: Proc Int Symp on Industrial Robots, Washington DC, pp. 135-152.

Wolkenhauer, O. \& Edmunds, J.M. (1997). A critique of fuzzy logic in control, Int J Electrical Engineering Education, Vol. 34(3), pp. 235-242.

Zhang, G. \& Hemami, A. (1997). An Overview of Robot Force Control, Robotica, Vol. 15, pp. 473-482. 


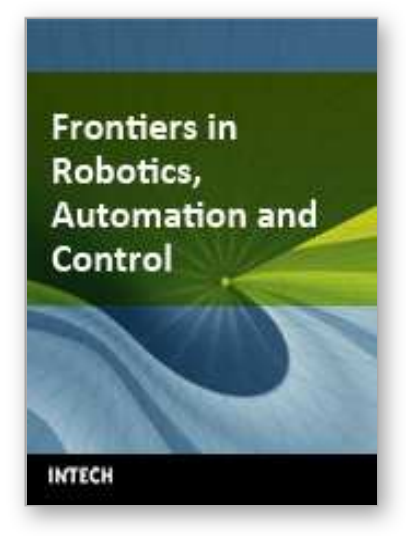

\author{
Frontiers in Robotics, Automation and Control \\ Edited by Alexander Zemliak
}

ISBN 978-953-7619-17-6

Hard cover, 450 pages

Publisher InTech

Published online 01, October, 2008

Published in print edition October, 2008

This book includes 23 chapters introducing basic research, advanced developments and applications. The book covers topics such us modeling and practical realization of robotic control for different applications, researching of the problems of stability and robustness, automation in algorithm and program developments with application in speech signal processing and linguistic research, system's applied control, computations, and control theory application in mechanics and electronics.

\title{
How to reference
}

In order to correctly reference this scholarly work, feel free to copy and paste the following:

Michael Short (2008). Evaluation of Robotic Force Control Strategies Using an Open Architecture Test Facility, Frontiers in Robotics, Automation and Control, Alexander Zemliak (Ed.), ISBN: 978-953-7619-17-6, InTech, Available from:

http://www.intechopen.com/books/frontiers_in_robotics_automation_and_control/evaluation_of_robotic_force_ control_strategies_using_an_open_architecture_test_facility

\section{INTECH}

open science | open minds

\section{InTech Europe}

University Campus STeP Ri

Slavka Krautzeka 83/A

51000 Rijeka, Croatia

Phone: +385 (51) 770447

Fax: +385 (51) 686166

www.intechopen.com

\section{InTech China}

Unit 405, Office Block, Hotel Equatorial Shanghai

No.65, Yan An Road (West), Shanghai, 200040, China

中国上海市延安西路65号上海国际贵都大饭店办公楼 405 单元

Phone: +86-21-62489820

Fax: $+86-21-62489821$ 
(C) 2008 The Author(s). Licensee IntechOpen. This chapter is distributed under the terms of the Creative Commons Attribution-NonCommercialShareAlike-3.0 License, which permits use, distribution and reproduction for non-commercial purposes, provided the original is properly cited and derivative works building on this content are distributed under the same license. 